\title{
Riesgos Psicosociales y Salud Laboral en Centros de Salud
}

\section{PSICOSOCIAL RISK AND OCCUPATIONAL HEALTH IN HEALTH CENTERS}

\author{
Nelson P. Castro Méndez
}

1. Universidad Arturo Prat, Facultad de Ciencias de la Salud, Psicólogo, Magister en Dirección y Gestión de Recursos Humanos, Académico Carrera de Psicología.

\section{RESUMEN}

Este estudio tuvo por objetivo describir la salud y el riesgo psicosocial en tres centros de salud públicos y analizar sus asociaciones con condiciones de trabajo y enfermedad. El método incluyó un diseño observacional, analítico, transversal y una muestra total de 438 funcionarios quienes respondieron el cuestionario validado en Chile, SUSESO/ISTAS 21. Los resultados muestran que 14,6\% reportan tener una enfermedad diagnosticada provocada y/o agravada por el trabajo lo que es consistente con bajos niveles de salud general, salud mental, vitalidad y altos niveles de síntomas de estrés y con condiciones de trabajo de desequilibrio entre esfuerzo-recompensa. Se observaron 4 riesgos psicosociales altos, comunes y presentes en todos los centros y 9 riesgos psicosociales altos que permiten discriminar entre funcionarios sanos y enfermos. Se discute y concluye la importancia de atender a los riesgos y condiciones comunes en centros de salud.

(Castro N, 2018. Riesgos Psicosociales y Salud Laboral en Centros de Salud. Cienc Trab. Sep-Dic; 20 [63]: 155-159).

Palabras clave: SALUD LABORAL, RIESGOS LABORALES, FACTORES DE RIESGO, CENTROS DE SALUD.

\section{ABSTRACT}

The objective of this study was to describe health and psychosocial risk in three public health centers and analyze their associations with working conditions and illness. The method included an observational, analytical, cross-sectional design and a total sample of 438 officials who answered the questionnaire validated in Chile, SUSESO / ISTAS 21. The results show that 14.6\% report having a diagnosed illness caused and / or aggravated by work, which is consistent with low levels of general health, mental health, vitality and high levels of stress symptoms and with imbalanced work conditions between effort-reward. There were 4 high psychosocial risks, common and present in all centers, and 9 high psychosocial risks that allow discriminating between healthy and sick officials. The importance of attending to common risks and conditions in health centers is discussed and concluded.

Key words: OCCUPATIONAL HEALTH, OCCUPATIONAL RISKS, RISK FACTORS, HEALTH CENTERS

\section{INTRODUCCION}

Las condiciones y características del trabajo han cambiado en la actualidad producto de la globalización y la consecuente expansión del mercado de servicios. La Organización moderna es dinámica, variable y tiene capacidad de cambio para responder a la fluctuación en la demanda, pero esta flexibilidad trae consigo inseguridad, incertidumbre y turbulencias que afectan al trabajador. Aun cuando el trabajo históricamente ha sido un riesgo para la salud, en nuestros días las condiciones presentes en la situación laboral, tales como la forma en que se organiza el trabajo y el contenido de la tarea son factores psicosociales de riesgo que afectan la salud del trabajador. Cuando estas situaciones laborales tienen una alta probabilidad de dañar gravemente la salud del trabajador se habla de riesgos psicosociales del

\section{Correspondencia / Correspondence:}

Sr. Nelson P. Castro Méndez

Telefóno: +5645-288913012

Victoria, Chile.

e-mail: nelcastr@unap.cl

Recibido: 10 agosto de 2018 / Aceptado: 13 de octubre de 2018 trabajo. ${ }^{1}$ Para Nieto ${ }^{2}$ las enfermedades causadas por las malas condiciones de trabajo son de las peores pandemias que castigan a la humanidad. En el 2014 se diagnosticaron 4.411 enfermedades profesionales en la mutualidades privadas en Chile, que representa una tasa de 0,09 enfermedades profesionales por cada 100 trabajadores. $^{3}$ La enfermedad relacionada con el trabajo es consecuencia de un factor individual de predisposición constitucional y un factor profesional que despliega una influencia favorecedora en relación con las condiciones de trabajo. ${ }^{4}$ Entre los efectos provocados por los factores psicosociales se encuentran alteraciones en la salud mental como ansiedad-insomnio y problemas psicosomáticos. ${ }^{5}$ Los profesionales que realizan su trabajo en el ámbito sanitario constituyen una población especialmente vulnerable a los riesgos derivados de su trabajo. ${ }^{6}$ En estos espacios el clima organizacional se ve afectado por la disposición de equipos y la distribución de personas y material como observaron Bustamante, Grandón y Lapo ${ }^{7}$, en un estudio que caracterizó el clima en dos hospitales de alta complejidad. Otro estudio en trabajadores de la salud pública en Chile encontró que en los técnicos la percepción de calidad de vida laboral es inferior a los directivos y que la calidad de vida laboral se relaciona al sindrome de burnout, evidenciando una relación inversa con el agotamiento emocional y la despersonalización. ${ }^{8}$ Por su parte Vidal et $\mathrm{al}^{9}$ encontraron que la calidad de vida del personal de salud, que consideró la motivación intrínseca, la carga de trabajo se relacionó positivamente con el ausentismo y que el 42,3\% presentó 
ausentismo por licencia médica observándose una mediana de una licencia por persona y un total de 15 días. Ansoleaga ${ }^{10}$ estudió indicadores de salud mental asociados a riesgo psicosocial laboral en un hospital público encontrando que 25\% de los trabajadores (muestra fue de 782 funcionarios de un hospital pediátrico de alta complejidad) usó drogas psicotrópicas, 34\% presentaba niveles altos de distrés, 23\% tuvo síntomas depresivos y que estos resultados estaban asociados en un $48 \%$ al reporte de baja latitud decisional, 41\% al bajo soporte social y $67 \%$ al desbalance entre esfuerzo y recompensa. Ansoleaga ${ }^{10}$ sugiere seguir investigando el trabajo en salud con atención especial a la dimensión de género y a las condiciones de trabajo en estos centros.

Esta investigación se propuso describir la salud y el riesgo psicosocial en Centros de Salud y analizar sus asociaciones con condiciones de trabajo y enfermedad

\section{MATERIAL Y MÉTODOS}

\section{Diseño, Procedimiento e Instrumento del estudio}

Para cumplir con el propósito del estudio se consideró un diseño observacional, analítico y transversal que incluyó la participación de tres centros de salud pública de la provincia de Malleco de la Región de la Araucanía, Chile; C1 $(n=95)$, C2 $(n=113)$ y C3 $(n=230)$. La muestra total quedó constituida por 438 funcionarios correspondiendo a un $88 \%$ del total de funcionarios. El Instrumento utilizado fue el Cuestionario SUSESO/ISTAS 21 versión completa, validado para población chilena por la escuela de Salud pública de la Universidad de Chile en conjunto con la Superintendencia de Seguridad Social ${ }^{11}$; el cual fue respondido en marzo-abril 2017. El cuestionario SUSESO/ISTAS 21 consta de 142 preguntas, entre las cuales hay 53 preguntas sobre salud, empleo, trabajo, salario, endeudamiento, ausentismo y 89 preguntas que miden riesgo psicosocial, agrupadas en 5 dimensiones: Exigencias psicológicas, Trabajo activo y posibilidades de desarrollo, Apoyo social en la empresa y calidad de liderazgo, Compensaciones, Doble presencia y en 19 subdimensiones: Exigencias psicológicas cuantitativas (CU), Exigencias psicológicas cognitivas (CO), Exigencias psicológicas emocionales (EM), Exigencias psicológicas de esconder emociones (EE), Exigencias psicológicas sensoriales (ES), Influencia (IN), Control sobre el tiempo de trabajo (CT), Sentido del trabajo (ST), Integración en la empresa (IE), Claridad de rol (RL), Conflicto de rol (CR), Calidad de liderazgo (CL), Calidad de relación son superiores (RS), Calidad de relación con compañeros de trabajo (RC), Estima (ET), Inseguridad respecto del contrato de trabajo (IC), Inseguridad respecto a las características específicas del trabajo (IT), Preocupación por tareas domésticas (DP). Los ítems tienen un formato de escala Likert 1-5 que va de Siempre a Nunca y contempla algunos items de orden inverso, según Manual SUSESO/ISTAS 21; 2016.

Se consideraron como variables de estudio: sexo, edad, dimensiones y subdimensiones de riesgo psicosocial, indicadores de salud y bienestar personal como salud general, salud mental, vitalidad, nivel de estrés, riesgos psicosociales, aspectos del trabajo y empleo.

\section{Análisis Estadísticos}

Para cada centro de salud se realizó un análisis descriptivo a fin de obtener frecuencias y las prevalencias de riesgos psicosociales altos (mayores a 50\%), se calcularon medias y porcentajes a fin de obtener los indicadores de salud: Salud General (SG), Salud
Mental (SM), Vitalidad (VT), Síntomas de Estrés (SR), licencias médicas, accidentes, enfermedad. Se consideró como enfermedad operacionalmente aquellos casos que reportaron tener una enfermedad diagnosticada provocada y/o agravada por el trabajo, aspecto medido dicotómicamente (sí/no) por el instrumento, luego se comparó si había diferencias significativas entre funcionarios sanos y enfermos en indicadores de salud entre los centros de salud. Para profundizar el análisis se tomó la muestra total $(n=438)$ distinguiendo dos grupos, uno de funcionarios sanos y otro de funcionarios enfermos y se estudió la relación de la enfermedad con variables de riesgo psicosocial y aspectos del trabajo y empleo, para lo cual se recurrió a tablas de contingencia con la respectiva significación estadística usando la prueba chi cuadrado.

\section{RESULTADOS}

\section{Descripción de la Muestra y Confiabilidad de los Instrumentos}

La muestra fue de tipo accidental y quedó constituida por tres centros de salud públicos $\mathrm{C} 1, \mathrm{C} 2$ y $\mathrm{C} 3$ que estaban disponibles y que contestaron el instrumento considerado en esta investigación en el lapso de marzo-abril 2017. Se obtuvo la Confiabilidad de los instrumentos para cada centro, el que mostró un Alpha de Cronbach de 0,716 para C1, de 0,794 para C2 y de 0,881 para C3. A su vez, las escalas de salud; SG, SM, VT, SR también lograron niveles adecuados de confiabilidad para los distintos centros, mostrando un promedio de 0,84 para $\mathrm{C} 1,0,83$ para $\mathrm{C} 2$ y 0,85 para C3, siendo la menor confiabilidad de 0,773 para SG del C2.

En la Tabla 1 puede observarse como quedó constituida la muestra. La Tabla 1 permite observar que en dos de los tres centros de salud el porcentaje de mujeres es mayor al de los hombres, la edad mayormente representada es el intervalo de los 26 a los 35 años, el estamento más representado es el de profesionales y el tipo de contrato mayoritario es el de Contrata (plazo fijo anual).

Tabla 1.

Descripción de la muestra.

\begin{tabular}{|c|c|c|c|c|c|c|}
\hline \multirow[b]{3}{*}{ Total } & \multicolumn{3}{|c|}{ C1 $(n=95)$} & \multicolumn{2}{|c|}{ C3 $(n=230)$} & \multirow{3}{*}{$\mathrm{H} n(\%)$} \\
\hline & $\mathrm{M} n(\%)$ & $\mathrm{H}$ n(\%) & $\mathrm{M} n(\%)$ & H n(\%) & $\mathrm{M} \mathrm{n}(\%)$ & \\
\hline & $55(58)$ & $40(42)$ & $75(66)$ & $38(34)$ & $110(48)$ & \\
\hline \multicolumn{7}{|l|}{ Rangos de edad } \\
\hline Menos de 26 & $2(4)$ & $3(7)$ & $6(8)$ & $3(8)$ & $14(13)$ & $11(9)$ \\
\hline $26-35$ & $26(47)$ & $16(40)$ & $28(37)$ & $19(50)$ & $55(50)$ & $42(35)$ \\
\hline $36-45$ & $12(22)$ & $8(20)$ & $23(31)$ & $4(11)$ & $27(24)$ & $31(26)$ \\
\hline $46-55$ & 10 (18) & $6(15)$ & $12(16)$ & 7 (18) & $10(9)$ & $23(19)$ \\
\hline Más de 55 & $5(9)$ & $7(18)$ & 6 (8) & $5(13)$ & $4(4)$ & $13(11)$ \\
\hline \multicolumn{7}{|c|}{ Participación por estamentos } \\
\hline Administrativos & $8(15)$ & $6(15)$ & $16(21)$ & 1 (2) & $29(26)$ & $8(7)$ \\
\hline Auxiliares & 5 (9) & $14(35)$ & $5(7)$ & $12(32)$ & $1(1)$ & $21(17)$ \\
\hline Profesionale & $21(38)$ & $12(30)$ & $24(32)$ & $16(42)$ & 60 (55) & $71(59)$ \\
\hline Técnicos & $21(38)$ & $8(20)$ & $30(40)$ & $9(24)$ & $20(18)$ & $18(15)$ \\
\hline Directivos & $0(0)$ & $0(0)$ & $0(0)$ & $0(0)$ & $0(0)$ & $2(2)$ \\
\hline \multicolumn{7}{|l|}{ Tipo Contrato } \\
\hline Titular (indefinido) & $16(29)$ & $14(35)$ & $23(31)$ & $11(29)$ & $9(8)$ & $13(11)$ \\
\hline $\begin{array}{l}\text { Contrata } \\
\text { (plazo fijo anual) }\end{array}$ & 26 & 20 & 40 & $23(61)$ & 70) & 73 \\
\hline Honorarios & $12(22)$ & $6(15)$ & $10(13)$ & $4(10)$ & $20(18)$ & $29(24)$ \\
\hline Otro & $1(2)$ & $0(0)$ & $2(3)$ & $0(0)$ & $4(4)$ & $5(4)$ \\
\hline
\end{tabular}

(M) Mujeres; (H) Hombres 
Tabla 2.

Prevalencia de riesgo psicosocial alto en funcionarios por centro salud.

\begin{tabular}{lccc} 
Prevalencia Riesgo Psicosocial Alto & C1(\%) & C2(\%) & C3(\%) \\
\hline Exigencias Psicológicas & 34,38 & 38,6 & 40 \\
Trabajo activo y posibilidades de desarrollo & 19,79 & 14,04 & 24,78 \\
Apoyo social en la empresa y calidad de Liderazgo & 27,08 & 24,56 & 40,87 \\
Compensaciones & 21,88 & 25,44 & 36,09 \\
Doble presencia & $\mathbf{5 9 , 3 8}$ & $\mathbf{4 8 , 2 5}$ & $\mathbf{5 3 , 0 4}$
\end{tabular}

Valores en negrita indican prevalencia $>50 \%$

Fuente: Elaboración propia

\section{Riesgo Psicosocial: Dimensiones y Subdimensiones}

En la Tabla 2 se muestra la prevalencia de riesgo psicosocial alto de los centros de salud. Se puede observar que éste se concentra en Doble Presencia exhibiendo una prevalencia de riesgo alto mayor al 50\% en dos de los tres centros.

$\mathrm{Al}$ examinar por subdimensiones de riesgo psicosocial dónde se genera la prevalencia más alta de riesgo para estos centros de salud, es posible observar aspectos comunes tal como lo muestra la tabla 3; las Exigencias psicológicas emocionales (EM), las Exigencias psicológicas de Esconder emociones (EE), las Exigencias psicológicas sensoriales (ES) y el margen de Influencia (IN) aparecen reiteradamente en todos los centros, indicando una alta prevalencia de riesgo en estas variables. Dos variables, la Claridad de rol (RL) y la Doble presencia (DP) exhiben una prevalencia alta de riesgo en dos de los tres centros de salud.

\section{Indicadores de Salud: Porcentaje de Accidentes, Licencias y Enfermedad}

Se obtuvo el porcentaje de accidentes, licencias médicas y de enfermedad relacionada al trabajo para cada centro. En el caso de los accidentes; El C2 presenta un mayor porcentaje de accidentabilidad de 8,8\% mientras que los otros centros presentan 5,3\% el C1 y 7,4\% el C3. En el caso de las licencias médicas el porcentaje es similar exhibiendo el C1 un 45,3\% de licencias, el C2 un 43,4\% y el C3 un $40,4 \%$ de licencias. En el caso de la percepción de enfermedad relacionada al trabajo el C1 muestra un porcentaje mayor de 16,8\%, seguido por el C2 con un 14,2\% y el C3 con un 13,9\% de personas que señalan tener una enfermedad diagnosticada provocada y/o agravada por el trabajo.

\section{Relación con Enfermedad}

A continuación, con el fin de estudiar las asociaciones de la enfermedad con condiciones de trabajo y riesgo psicosocial, se separaron los datos en dos grupos para cada centro, aquellos que señalan no tener una enfermedad o funcionarios sanos y aquellos que declaran tener una enfermedad relacionada al trabajo o funcionarios enfermos. Esto permitió obtener indicadores de Salud general (SG), Salud mental (SM), Vitalidad (VT) y Síntomas de estrés (SR) para ambos grupos, lo que es posible visualizar en la tabla 4
Tabla 4.

Indicadores de Salud General, Salud Mental, Vitalidad y Sintomas de Estrés en funcionarios sanos (Fs.) y enfermos (Fe.).

\begin{tabular}{lcccccc} 
Indicadores & \multicolumn{2}{c}{ C1 } & \multicolumn{2}{c}{ C2 } & \multicolumn{2}{c}{ C3 } \\
Salud & Fs. $(n=79)$ & $F e .(n=16)$ & Fs. $(n=97)$ & $F e .(n=16)$ & Fs. $(n=198)$ & $F e .(n=32)$ \\
SG & 71,45 & 60,00 & 75,41 & 73,43 & 71,33 & 65,31 \\
SM & 73,16 & $53,75^{*}$ & 77,47 & $60,31^{*}$ & 66,84 & 62,81 \\
VT & 63,76 & $48,04^{*}$ & 68,49 & $53,90^{*}$ & 61,17 & 56,64 \\
SR & 23,47 & $40,62^{*}$ & 24,09 & $33,20^{*}$ & 30,96 & $39,97^{*}$
\end{tabular}

${ }^{*} p<0,05$ Diferencia significativa Fs-Fe prueba no paramétrica U de Mann-Whitney. Fuente: Elaboración propia.

La tabla 4 muestra que aquellos que declaran tener una enfermedad relacionada al trabajo exhiben peores indicadores de salud que aquellos que se declaran sanos. Estas diferencias entre grupo enfermo y sano en los indicadores de salud son consistentes por centro; se muestran aquellas diferencias que son significativas (prueba no paramétrica U de Mann-Whitney; $\mathrm{p}<0,05$ ). $\mathrm{Al}$ considerar el total de la muestra $(n=438)$ las diferencias entre sanos y enfermos son significativas para todos los indicadores considerados. Por otra parte, la salud general reportada por hombres $(M=72,47 \quad D E=19,18)$ es mayor que la de las mujeres $(M=70,66 \quad D E=18,32)$, la salud mental en hombres $(M=73,76$ $\mathrm{DE}=20,24)$ es mayor que en mujeres $(M=65,66 \mathrm{DE}=20,71)$, la vitalidad reportada en hombres $(M=67,07 \mathrm{DE}=20,01)$ es mayor que en mujeres $(M=58,15 \mathrm{DE}=19,34)$ y el reporte de sintomas de estrés $(M=25,56 D E=19,05)$ es menor en los hombres que en las mujeres $(M=32,17 \mathrm{DE}=18,23)$.

$\mathrm{Al}$ estudiar los aspectos que están relacionados a enfermedad es posible observar que existen condiciones y riesgos psicosociales que permiten diferenciar al grupo de funcionarios enfermos del grupo de funcionarios sanos. En la tabla 5 se observan estos aspectos sociodemográficos y del trabajo en personas que declararon sí tener una enfermedad diagnosticada provocada y/o agravada por el trabajo. Los resultados muestran relación entre enfermedad con sexo mujer, con edad de 26-35 años, con accidentabilidad, con baja equivalencia entre lo trabajado y el sueldo percibido, con el cambio de horario que provoca inconvenientes al trabajador y con las licencias médicas.

Posteriormente, se estudió la relación de la enfermedad con los riesgos psicosociales y en la tabla 6 se observan los niveles de riesgo psicosocial significativos en personas que declararon sí tener una enfermedad diagnosticada provocada y/o agravada por el trabajo.

Los resultados mostraron relación sólo con 9 subdimensiones; con Exigencias psicológicas cuantitativas (CU), Exigencias psicológicas emocionales (EM), Exigencias psicológicas de esconder emociones (EE), Influencia (IN), Conflicto de rol (CR), Relación con superiores (RS), Relación con compañeros (RC), Estima (ET) y Doble presencia (DP).

Tabla 3.

Prevalencia de riesgo psicosocial alto (>50\%) en funcionarios por centro salud y por subdimensiones.

\begin{tabular}{|c|c|c|c|c|c|c|c|c|c|c|c|c|c|c|c|c|c|c|c|}
\hline & $\mathrm{CU}$ & $\mathrm{CO}$ & EM & $\mathrm{EE}$ & ES & IN & PD & CT & ST & IE & $\mathrm{RL}$ & CR & $\mathrm{CL}$ & RS & $\mathrm{RC}$ & ET & IC & IT & DP \\
\hline $\mathrm{C} 1$ & & & 54,17 & 58,33 & 63,54 & 55,21 & & & & & 51,04 & & & & & & & & 59,38 \\
\hline $\mathrm{C} 2$ & & & 54,39 & 54,39 & 57,02 & 58,77 & & & & & & & & & & & & & \\
\hline C3 & & & 59,13 & 54,78 & 56,09 & 51,74 & & & & & 55,22 & & & & & & & & 53,04 \\
\hline
\end{tabular}

Fuente: Elaboración propia 
Tabla 5.

Aspectos sociodemográficos y del trabajo relacionados a enfermedad.

\begin{tabular}{|c|c|c|}
\hline & n $(\%)$ & $2(g . l))$. \\
\hline \multicolumn{3}{|l|}{ Sexo } \\
\hline Hombre & $17(26,6 \%)$ & \\
\hline Mujer & $47(73,4 \%)$ & $10,517^{* *}(1)$ \\
\hline \multicolumn{3}{|l|}{ Edad } \\
\hline Menos de 26 & $1(1,6 \%)$ & \multirow{5}{*}{$10,242^{*}(4)$} \\
\hline $26-35$ & $37(57,8 \%)$ & \\
\hline $36-45$ & $11(17,2 \%)$ & \\
\hline $46-55$ & $9(14,1 \%)$ & \\
\hline Más de 55 & $6(9,4 \%)$ & \\
\hline \multicolumn{3}{|l|}{ Accidentes } \\
\hline No & $55(85,9 \%)$ & \multirow[b]{2}{*}{$5,053^{*}(1)$} \\
\hline Si & $9(14,1 \%)$ & \\
\hline \multicolumn{3}{|c|}{ Equivalencia trabajo-sueldo } \\
\hline Sí & $12(18,8 \%)$ & \multirow{4}{*}{$16,776^{* *}(3)$} \\
\hline No, trabajo superior & $26(40,6 \%)$ & \\
\hline No, trabajo inferior & $8(12,5 \%)$ & \\
\hline No sabe & $18(28,1 \%)$ & \\
\hline \multicolumn{3}{|l|}{ Cambio horario y aviso } \\
\hline No hay cambio & $43(67,2 \%)$ & \multirow{5}{*}{$10,107^{*}(4)$} \\
\hline Aviso, sin inconvenientes & $5(7,8 \%)$ & \\
\hline Aviso,con inconvenientes & $8(12,5 \%)$ & \\
\hline Aviso un dia antes & $5(7,8 \%)$ & \\
\hline Aviso mismo día & $3(4,7 \%)$ & \\
\hline \multicolumn{3}{|l|}{ Licencia } \\
\hline Sin licencia & $27(42,2 \%)$ & \\
\hline \multirow[t]{2}{*}{ Con licencia } & $37(57,8 \%)$ & $7,452^{*}(1)$ \\
\hline & ${ }^{*} p<0,05$ & ${ }^{* *} p<0,001$ \\
\hline
\end{tabular}

Tabla 6.

Riesgos psicosociales relacionados a enfermedad.

$\begin{array}{lcccc} & \text { Bajo } & \text { Medio } & \text { Alto } & \chi 2 \text { (g.I.) } \\ \text { CU } & 19(29,7 \%) & 11(17,2 \%) & 34(53,1 \%)^{*} & 11,952(2) \\ \text { EM } & 2(3,1 \%) & 13(20,3 \%) & 49(76,6 \%)^{*} & 13,066(2) \\ \text { EE } & 4(6,3 \%) & 11(17,2 \%) & 49(76,6 \%)^{* *} & 14,387(2) \\ \text { IN } & 2(3,1 \%) & 17(26,6 \%) & 45(70,3 \%)^{*} & 11,779(2) \\ \text { CR } & 21(32,8 \%) & 15(23,4 \%) & 28(43,8 \%)^{*} & 7,416(2) \\ \text { RS } & 15(23,4 \%) & 16(25 \%) & 33(51,6 \%)^{*} & 8,458(2) \\ \text { RC } & 9(14,1 \%) & 26(40,6 \%) & 29(45,3 \%)^{*} & 8,240(2) \\ \text { ET } & 9(14,1 \%) & 19(29,7 \%) & 36(56,3 \%)^{*} & 9,978(2) \\ \text { DP } & 2(3,1 \%) & 17(26,6 \%) & 45(70,3 \%)^{*} & 8,976(2) \\ { }^{*} \mathrm{p}<0,05 & { }^{* *} \mathrm{p}<0,001 & & & \end{array}$

\section{DISCUSIÓN}

La presente investigación tuvo por propósito describir la salud y el riesgo psicosocial en Centros de Salud y analizar sus asociaciones con condiciones de trabajo y enfermedad. Los resultados mostraron que al igual que en otras investigaciones en centros de salud $^{9,10}$ la proporción de mujeres es mayor y el tipo de contrato principal es Contrata.

Se observó la presencia de riesgos psicosociales de alta prevalencia que son comunes en los centros de salud y que exponen a los trabajadores a enfermar por condiciones de trabajo; entre ellos se encontró las Exigencias psicológicas emocionales (EM), el Esconder emociones (EE), las Exigencias psicológicas sensoriales (ES), el riesgo por Influencia (IN), la Claridad de rol (RL) y la Doble presencia (DP); todas variables que Alvarado et al $^{11}$ reportan correlacionadas significativa- mente con salud general (Cuestionario de Salud General GHQ-12). Se destacan en el estudio de Alvarado et al $^{11}$ entre las más altas EM $(\mathrm{r}=0,303 \mathrm{p}<0,001)$ y $\mathrm{EE}(\mathrm{r}=0,222 \mathrm{p}<0,001)$. Estos riesgos estarían, entonces, aportando a la relación con la enfermedad en los centros de salud de manera más estructural u organizacional, revistiendo gran interés investigar en ello. Algunos estudios han mostrado relación entre las exigencias psicológicas emocionales y el conflicto de rol en servicios de salud ${ }^{12}$ observándose en esta investigación como riesgo común la claridad de rol, fuente para el conflicto de rol (CR) que vemos efectivamente aparecer en los resultados de los funcionarios enfermos. Mansilla ${ }^{13}$ muestra la existencia de consecuencias en la salud mental del estrés de rol, una de cuyas fuentes es el riesgo por claridad de rol. En una revisión teórica $\mathrm{Grau}^{14}$ sobre los riesgos psicosociales y las enfermedades crónicas no transmisibles concluye la importancia de los estados emocionales mal manejados, el aislamiento social y la incomunicación. Los centros de salud son Organizaciones que involucran Trabajo Emocional (TE), constructo multidimensional referido a la carga emocional, la valencia, su variedad y relación con el puesto de trabajo. El TE no afecta negativamente al trabajador cuando lo siente parte de su identidad profesional, pero la disonancia emocional (expresión de emociones impuestas por normas que no representan los verdaderos sentimientos) puede ser una modalidad de conflicto de $\operatorname{rol}^{15} \mathrm{y}$ vincularse al desarrollo de la enfermedad. Estudios recientes indican que servicios de salud y de educación son las Organizaciones que más se asocian con el alto grado de exigencia emocional. ${ }^{16}$ Frente a las altas exigencias psicológicas encontradas en centros de salud y el estrés derivado de la ambigüedad de rol, resultan esperables conflictos de rol y problemas de relaciones interpersonales. Al respecto, GarcíaRodríguez et $\mathrm{al}^{17}$ estudiaron el entorno psicosocial y el estrés en trabajadores de la salud y concluyen que tener una buena relación social actúa como factor protector frente a la posibilidad de desarrollar sintomatología de estrés percibido y que las exigencias emocionales, el conflicto de rol y la inseguridad en el puesto de trabajo actuarían como factores de riesgo. Otros autores han encontrado asociaciones en trabajadores de la salud entre las altas exigencias psicológicas y el burnout. ${ }^{18}$

$\mathrm{Al}$ intentar comprender la relación con la enfermedad desde un nivel más individual, nueve son los riesgos psicosociales más asociados en trabajadores que reportan enfermedad. En el presente estudio se observó relación con las Exigencias psicológicas cuantitativas (CU), las Exigencias psicológicas emocionales (EM), el Esconder emociones (EE), la Influencia (IN), el Conflicto de rol (CR), la Relación con superiores (RS), la Relación con compañeros (RC), la Estima (ET) y la Doble presencia (DP). Estos riesgos podrían quedar expresados de forma resumida como altas exigencias en el trabajador enfermo (CU, EM, EE), bajo nivel de influencia (IN) y bajo soporte o apoyo (RS, RC, $\mathrm{CR}, \mathrm{ET})$, condiciones que según el modelo de Demanda-ControlApoyo $^{19}$ explicarían la enfermedad. Bajo estas mismas condiciones la investigación de Ansoleaga ${ }^{10}$ encontró 2,98 veces más posibilidades de sufrir sintomatología depresiva, 3,33 veces más posibilidades de presentar distrés elevado y 1,59 más veces de presentar consumo de psicotrópicos que quienes no están bajo dichas condiciones, lo que va en la misma dirección de lo encontrado en esta investigación. Otros autores ${ }^{20}$ han revisado 10 años de estudios en revistas de alto impacto sobre los importantes efectos (Odds ratio oscilan entre 1,3 y 1,7) de las condiciones adversas de trabajo como las altas exigencias y el bajo control y apoyo, en la enfermedad cardiovascular. Asimismo, informan de estudios que muestran una correlación de $\mathrm{r}=$ 0,42 entre las demandas emocionales y el burnout; entre demandas 
emocionales y depresión en mujeres. Las exigencias cuantitativas (CU) se relacionan significativamente con ausentismo, así lo mostró el estudio de Vidal et $\mathrm{al}^{9}$ en un centro de salud en el cual la carga de trabajo aumenta la probabilidad de ausentismo, lo que también se observa en este estudio.

Respecto de Indicadores de salud; se observó que el 14,6\% reporta alguna enfermedad relacionada y/o agravada por el trabajo de los cuales $26,6 \%$ son hombres contra $73,4 \%$ que son mujeres y que los hombres en la muestra total reportaron mejores indicadores de salud que las mujeres. Quienes refieren tener una enfermedad asociada al trabajo exhiben consistentemente problemas en su salud mental, en su vitalidad y reportan tener síntomas de estrés, lo que hace esperar trabajadores con problemas de ánimo, agotados y sin energía, posiblemente con problemas para dormir, de ansiedad y con dificultades para tomar decisiones apropiadas.

Vidal et $\mathrm{al}^{9}$, reportan un $42,3 \%$ de ausentismo por licencias médicas lo que coincidente con el presente estudio en donde se obtuvo un $43,03 \%$ en promedio y el cual estaría relacionado al reporte de enfermedad en un 57,8\%, a la falta de equilibrio entre el trabajo desempeñado y el sueldo recibido, al cambio en las condiciones de trabajo que causan inconvenientes y a los accidentes sufridos en los últimos doce meses. En la presente investigación no hubo relaciones significativas con otras variables como estamento, antigüedad, jornada de trabajo, tipo de contrato, endeudamiento. Cabe señalar que el presentismo o el ir a trabajar enfermo está asociado al deterioro del desempeño laboral autopercibido. ${ }^{21}$

Se concluye que el trabajo en centros de salud presenta de manera común importantes riesgos psicosociales con alta prevalencia en exigencias psicológicas y bajos niveles de influencia o latitud decisional. La claridad del rol es un riesgo psicosocial de alta prevalencia también presente y que podría estar desencadenando conflictos de rol resultando en un bajo soporte o apoyo social percibido por el traba- jador. Ambos aspectos permiten explicar los indicadores de salud encontrados como los altos niveles de licencias. La enfermedad en estos centros parece relacionarse al sexo, a la edad, a las condiciones de cambios imprevistos en horarios y que conllevan inconvenientes para el trabajador, a la percepción de desbalance o desequilibrio entre esfuerzo recompensa. También se asocia al desgaste emocional que involucra este tipo de trabajos con poco grado de influencia y baja claridad de rol lo que podría terminar afectando las relaciones con superiores y compañeros. Estos resultados aportan evidencia a las condiciones de trabajo en centros de salud públicos, destacan los aspectos comunes y su relación con la enfermedad, lo que permite orientar las intervenciones que deberian hacerse en ellos; atendiendo oportunamente al desgaste emocional y sus posibles consecuencias en las relaciones con superiores y compañeros, así como a las posibilidades de hacer pequeños cambios o influir en las condiciones de trabajo por parte del funcionario.

Las limitaciones de esta investigación están en que se consideró sólo tres centros, que la selección de los centros fue accidental siendo aquellos que contestaron el instrumento en el tiempo del estudio. Se sugiere profundizar el estudio de las variables que involucran desgaste o cansancio emocional en este tipo de Organizaciones con más centros y su relación con la enfermedad profesional.

\section{Agradecimientos}

Se agradece la colaboración y disposición de los funcionarios del Instituto de Seguridad Laboral (ISL) de la región de la Araucanía por el apoyo a esta investigación.

Fuente de Financiamiento: Esta investigación fue realizada en el contexto de Proyectos de Investigación concursables Internos 2015, financiado por la Universidad Arturo Prat.

\section{REFERÊNCIAS}

1. Moreno B. Factores y riesgos laborales psicosociales: conceptualización, historia y cambios actuales. Med Segur Trab. 2011; 57 Supl 1:4-19.

2. Nieto J. Enfermedades laborales, una pandemia que requiere prevención. Med Segur Trab 2014; 60(234):1-3.

3. Almonte J, Mena C, Ortiz S, Osorio J. Psiquiatria y ley de enfermedades profesionales en Chile: revisión histórica y crítica de una relación compleja. Rev Med Chile. 2016; 144(12):1591-1597.

4. Mur de Viu C, Maqueda J. Salud laboral y salud mental: estado de la cuestión. Med Segur Trab. 2011; 57 supl 1:1-3.

5. Vásquez A, Pérez $D$, Moreno R, Arroyo $F$, Suárez J. Factores psicosociales motivacionales y estado de salud. Med Segur Trab. 2010; 56(218):12-21.

6. Morillejo $\mathrm{E}_{1}$ Pozo C. Análisis de los factores psicosociales de riesgo en los profesionales dedicados al cuidado de la salud. Rev Psicol Trab Organ. 2001; 17(3):273-294.

7. Bustamante-Ubilla $M$, Grandón $M$, Avendaño, Lapo M. Caracterización del clima organizacional en hospitales de alta complejidad en Chile. Estudios Gerenciales. 2015; 31(137):432-440.

8. Pérez-Zapata D, Zurita R. Calidad de vida laboral en trabajadores de salud pública en Chile. Salud Sociedad. 2014; 5(2):172-180.

9. Vidal C, Palavecino I, Moya P, Toro C, Huerta1, Hoffmeister L. Calidad de vida del personal de salud y su relación con el ausentismo. Cienc Trab. 2017; 19(60):188-193.

10. Ansoleaga E. Indicadores de salud mental asociados a riesgo psicosocial laboral en un hospital público. Rev Méd Chile. 2015; 143(1):47-55.11. Alvarado R, Pérez-Franco J, Saavedra N, Fuentealba C, Alarcón A, Marchetti N, Aranda W. Validación de un cuestionario para evaluar riesgos psicosociales en el ambiente laboral en Chile. Rev Méd Chile 2012; 140(9):1154-1163.

12. Quiñones M, Tapia T, Díaz C. El rol de las demandas y los recursos laborales en la salud mental de los trabajadores chilenos. Cienc Trab. 2012: 14(45):201-210.

13. Mansilla F. Consecuencias del estrés de rol. Med Segur Trab 2011; 57(225):361370.

14. Grau J. Enfermedades crónicas no transmisibles: un abordaje desde los factores psicosociales. Salud Sociedad. 2016; 7(2):138-166.

15. Moreno-Jiménez B, Gálvez M, Rodríguez-Carvajal R, Garrosa E. Emociones y salud en el trabajo: análisis del constructo "trabajo emocional" y propuesta de evaluación. Rev Latinoam Psicol. 2010; 42(1):63-73.

16. Marrero J. Las exigencias emocionales en el trabajo. El caso español. Pap Rev Sociol. 2015; 100(2):173-193.

17. García-Rodríguez A, Gutiérrez-Bedmara M. Bellón-Saameño J, Muñoz-Bravo C, Fernández-Crehuet J. Entorno psicosocial y estrés en trabajadores sanitarios de la sanidad pública: diferencias entre atención primaria y hospitalaria. Aten Primaria. 2015; 47(6):359-366.

18. Pastorino MS, Alvarado R. Factores psicosociales del trabajo y síndrome de burnout en trabajadores de la salud. Rev Salud Pública (Córdoba). 2008; 12(2):6-13.

19. Karasek R, Theorell T. Healthy work: stress, productivity and the reconstruction of the working life. New York: Basic Books; 1990.

20. Vieco GF, Abello R. Factores psicosociales de origen laboral, estrés y morbilidad en el mundo Psicol Caribe. 2014; 31(2):354-385.

21. Vera-Calzaretta A, Carrasco-Dájer C, Da Costa S, Páez-Rovira D. Factores psicosociales del presentismo en trabajadores del sistema de salud chileno. Rev Psicol Trab Organ. 2015; 31(2):119-128. 